\title{
Exponential Stability of Periodic Solutions for Cohen-Grossberg Neural Networks with Continuously Distributed Delays
}

\author{
Yonggui Kao ${ }^{1,2}$ Qinghe Ming ${ }^{1}$ Cunchen Gao ${ }^{2}$ \\ ${ }^{1}$ Department of Mathematics, Zaozhaung University, Zaozhaung 277160, P.R. China \\ ${ }^{2}$ Department of Mathematics,Ocean University of China, Qingdao 266071, P.R.China
}

\begin{abstract}
A class of Cohen-Grossberg neural networks with distributed delays are considered. By using the coincidence degree theorem and differential inequality techniques, sufficient conditions for the existence and exponential stability of the periodic solutions are established, Without assuming the boundedness, monotonicity, and differentiability of activation functions and any symmetry of interconnections. The results of this paper are new.
\end{abstract}

Keywords: Cohen-Grossberg neural networks, Periodic solution, Exponential stability, Coincidence degree theory, Distributed delays

\section{Introduction}

Cohen and Grossberg neural networks (CGNNs) model, first introduced by Cohen and Grossberg in [1], have been widely investigated for the sake of theoretical interest as well as application considerations. Many good results have already been obtained by some authors in [2]-[15], and have been widely applied within various engineering and scientific fields such as neuro-biology, population biology, and computing technology. But to the best of our knowledge, the existing literature dealing with unbounded activation functions and distributed delays appears to be scarce. In fact, neural networks usually has a spatial extent due to the presence of an amount of parallel pathways with a variety of axon sizes and lengths. Thus, the delays in artificial neural networks are usually continuously distributed [14]-[20]. Moreover, in many applications, the properties of periodic oscillatory solutions and global exponential stability are of great interest. For example, the human brain is in a periodic oscillatory or chaos state, hence it is of prime importance to study periodic oscillation, global exponential stability and chaos phenomenon of neural networks. This motivates us consider the following CGNNs with distributed delays

$$
\begin{gathered}
\dot{x}_{i}(t)=-a_{i}\left(t, x_{i}(t)\right)\left[b_{i}\left(t, x_{i}(t)\right)-\sum_{j=1}^{n} c_{i j}(t) f_{j}\left(x_{j}(t)\right)\right. \\
\left.-\sum_{j=1}^{n} d_{i j}(t) f_{j}\left(\int_{0}^{\infty} K_{i j}(u) x_{j}(t-u) d u\right)+I_{i}(t)\right], \\
i=1,2, \cdots, n,
\end{gathered}
$$

where $a_{i}$ and $b_{i}$ are continuous functions on $R^{2}$, $f_{j}, c_{i j}, d_{i j}$ and $I_{i}$ are continuous functions on $R ; n$ corresponds to the number of units in a neural network; $x_{i}(t)$ denotes the potential (or voltage) of cell $i$ at time $t ; a_{i}$ represents an amplification function; $b_{i}$ is an appropriately behaved function; $c_{i j}(t)$ and $d_{i j}(t)$ denote the strengths of connectivity between cell $i$ and $j$ at time $t$ respectively. The activation function $f_{i}(\cdot)$ shows how the $i$ th neuron reacts to the input; $K_{i j}(t) \geq 0$ corresponds to the time delay required in processing and transmitting a signal from the $j$ th cell to the $i$ th cell at time $t$, and $I_{i}(t)$ denotes the $i$ th component of an external input source introduced from outside the network to cell $i$ at time $t, i, j=1,2, \ldots, n$.

Throughout this paper, it will be assumed that

$\left(H_{1}\right) \quad c_{i j}, d_{i j}, I_{i}: R \rightarrow R$ are periodic functions with a common period $\omega(>0), a_{i}$ and $b_{i}$ are $\omega$-periodic in the first variable, where $i, j=$ $1,2, \ldots, n$.

$\left(H_{2}\right)$ For $i=1,2, \ldots, n$, there exist positive constants $\underline{a_{i}}$ and $\overline{a_{i}}$ such that

$$
\underline{a_{i}}<a_{i}(t, u)<\overline{a_{i}}, \quad \text { for all } t, u \in R .
$$

$\left(H_{3}\right)$ For $i=1,2, \ldots, n$, there exist positive constants $\underline{b}_{i}$ and $\bar{b}_{i}$ such that

$$
0 \leq u b_{i}(t, u), \quad \underline{b}_{i}|u| \leq\left|b_{i}(t, u)\right| \leq \bar{b}_{i}|u|,
$$

for all $t, u \in R$. 
$\left(H_{4}\right)$ For $i=1,2, \ldots, n$, there exist non-negative constants $L_{j}$ such that

$$
\left|f_{j}(u)-f_{j}(v)\right| \leq L_{j}|u-v| \text { for all } u, v \in R .
$$

$\left(T_{5}\right)$ For each $i \in\{1,2, \cdots, n\}, j \in$ $\{1,2, \cdots, n\}$, the delay kernels $K_{i j}:[0, \infty) \rightarrow R$ are continuous, integrable and there exit nonnegative constants $k_{i j}$ such that

$$
\int_{0}^{\infty}\left|K_{i j}(s)\right| d u \leq k_{i j}
$$

$\left(T_{6}\right)$ For each $i \in\{1,2, \cdots, n\}, j \in$ $\{1,2, \cdots, n\}$, there exists a constant $\lambda_{0}$ such that

$$
\int_{0}^{\infty}\left|K_{i j}(s)\right| \exp \left(\lambda_{0} s\right) d s<+\infty .
$$

For convenience, we introduce some notations. We can choose constants $\tau, \overline{c_{i j}}, \overline{d_{i j}}$ and $\overline{I_{i}}$ such that

$$
\begin{gathered}
\overline{c_{i j}}=\max _{t \in[0, \omega]}\left|c_{i j}(t)\right|, \overline{d_{i j}}=\max _{t \in[0, \omega]}\left|d_{i j}(t)\right|, \\
\overline{I_{i}}=\max _{t \in[0, \omega]}\left|I_{i}(t)\right|,
\end{gathered}
$$

We will use $x=\left(x_{1}, x_{2}, \cdots, x_{n}\right)^{T} \in R^{n}$ to denote a column vector, in which the symbol $\left({ }^{T}\right)$ denotes the transpose of a vector. For matrix $D=\left(d_{i j}\right)_{n \times n}, D^{T}$ denotes the transpose of $D$, and $E_{n}$ denotes the identity matrix of size $n$. A matrix or vector $D \geq 0$ means that all entries of $D$ are greater than or equal to zero. $D>0$ can be defined similarly. For matrices or vectors $D$ and $E$, $D \geq E$ (resp. $D>E$ ) means that $D-E \geq 0$ (resp. $D-E>0)$. For $V(t) \in C((a,+\infty), R)$, let

$$
\begin{gathered}
D^{-} V(t)=\limsup _{h \longrightarrow 0^{-}} \frac{V(t+h)-V(t)}{h}, \\
D_{-} V(t)=\liminf _{h \longrightarrow 0^{-}} \frac{V(t+h)-V(t)}{h}, \forall t \in(a,+\infty) .
\end{gathered}
$$

As usual, we introduce the phase space $C\left((-\infty, 0] ; R^{n}\right)$ as a Banach space of continuous mappings from $(-\infty, 0]$ to $R^{n}$ equipped with the supremum norm defined by

$$
\|\varphi\|=\max _{1 \leq i \leq n} \sup _{-\infty \leq t \leq 0}\left|\varphi_{i}(t)\right|
$$

for all $\varphi=\left(\varphi_{1}(t), \quad \varphi_{2}(t), \quad \cdots, \varphi_{n}(t)\right)^{T} \in$ $C\left((-\infty, 0] ; R^{n}\right)$.

The initial conditions associated with system (1.1) are of the form

$$
x_{i}(s)=\varphi_{i}(s), s \in(-\infty, 0], i=1,2, \cdots, n,
$$

where $\varphi=\left(\varphi_{1}(t), \varphi_{2}(t), \cdots, \varphi_{n}(t)\right)^{T} \in$ $C\left((-\infty, 0] ; R^{n}\right)$.

Definition 1. Let $Z^{*}(t)=\left(x_{1}^{*}(t), x_{2}^{*}(t), \cdots\right.$, $\left.x_{n}^{*}(t)\right)^{T}$ be an $\omega$-periodic solution of system (1.1) with initial value $\varphi^{*}=\left(\varphi_{1}^{*}(t), \varphi_{2}^{*}(t), \cdots, \varphi_{n}^{*}(t)\right)^{T}$ $\in C\left((-\infty, 0] ; R^{n}\right)$. If there exist constants $\alpha>0$ and $M>1$ such that for every solution $Z(t)=$ $\left(x_{1}(t), x_{2}(t), \cdots, x_{n}(t)\right)^{T}$ of system (1.1) with any initial value $\varphi \in C\left((-\infty, 0] ; R^{n}\right),\left|x_{i}(t)-x_{i}^{*}(t)\right| \leq$ $M\left\|\varphi-\varphi^{*}\right\| e^{-\alpha t}, \forall t>0, \quad i=1,2, \cdots, n$. Then $Z^{*}(t)$ is said to be global exponential stable.

Definition 2. A real $n \times n$ matrix $H=\left(h_{i j}\right)$ is said to be an $M$-matrix if $h_{i j} \leq 0, i, j=$ $1,2, \cdots, n, i \neq j$, and $H^{-1} \geq 0$.

\section{Preliminaries}

First, consider an abstract equation in a Banach space $X$,

$$
L x=\lambda N x, \quad \lambda \in(0,1),
$$

where $L: D o m L \cap X \rightarrow X$ is a linear operator and $\lambda$ is a parameter. Let $P$ and $Q$ denote two projectors, $P: \operatorname{Dom} L \cap X \rightarrow K e r L$ and $Q: X \rightarrow X / I m L$. For convenience, we introduce a continuation theorem [23] as follows.

Lemma 2.1.[23] Let $X$ be a Banach space. Suppose that $L: D o m L \subset X \longrightarrow X$ is a Fredholm operator with index zero and $N: \bar{\Omega} \longrightarrow X$ is $L$-compact on $\bar{\Omega}$ with $\Omega$ open bounded in $X$. Moreover, assume that all the following conditions are satisfied.

(1) $L x \neq \lambda N x, \forall x \in \partial \Omega \cap \operatorname{DomL}, \lambda \in(0,1)$;

(2) $Q N x \neq 0, \forall x \in \partial \Omega \cap \operatorname{KerL}$;

(3) $\operatorname{deg}_{B}\{Q N, \Omega \cap K \operatorname{Ker} L, 0\} \neq 0, \operatorname{deg}_{B}$ denotes the Brouwer degree. Then equation $L x=N x$ has at least one solution in $\bar{\Omega}$.

For ease of exposition, throughout this paper we will adopt the following notations:

$$
\begin{gathered}
\left|x_{i}\right|_{\infty}=\max _{t \in[0, \omega]}\left|x_{i}(t)\right|, u(t)=\left(x_{1}(t), x_{2}(t), \cdots,\right. \\
\left.x_{n}(t)\right)^{T},\left|x_{i}\right|_{k}=\left(\int_{0}^{\omega}\left|x_{i}(t)\right|^{k} d t\right)^{1 / k}, i=1,2, \cdots, n .
\end{gathered}
$$

We denote $X$ as the set of all continuously $\omega$-periodic functions $u(t)$ defined on $R$, and denote $\|u\|_{X}=\max \left\{\left|x_{1}\right|_{\infty},\left|x_{2}\right|_{\infty}, \cdots,\left|x_{n}\right|_{\infty}\right\}$. Then, $X$ is a Banach space when it is endowed with the norm $\|u\|_{X}$. Let for $u(t)=\left(x_{1}(t), x_{2}(t), \cdots, x_{n}(t)\right)^{T} \in$ $X$,

$$
(N u)_{i}(t)=-a_{i}\left(t, x_{i}(t)\right)\left[b_{i}\left(t, x_{i}(t)\right)-\sum_{j=1}^{n} c_{i j}(t)\right.
$$




$$
\begin{gathered}
\times f_{j}\left(x_{j}(t)\right)-\sum_{j=1}^{n} d_{i j}(t) f_{j}\left(\int_{0}^{\infty} K_{i j}(u) x_{j}(t-u) d u\right) \\
\left.+I_{i}(t)\right], \quad i=1,2, \cdots, n, \quad(2.2) \\
(L u)(t)=u^{\prime}(t)=\left(x_{1}^{\prime}(t), x_{2}^{\prime}(t), \cdots, x_{n}^{\prime}(t)\right)^{T}, \\
D o m L=\left\{u(t): u(t) \in X, u^{\prime}(t) \in X\right\}, \quad(2.3) \\
P u=Q u=\frac{1}{\omega} \int_{0}^{\omega} u(t) d t=\left(\frac{1}{\omega} \int_{0}^{\omega} x_{1}(t) d t,\right. \\
\left.\frac{1}{\omega} \int_{0}^{\omega} x_{2}(t) d t, \cdots, \frac{1}{\omega} \int_{0}^{\omega} x_{n}(t) d t\right)^{T} .
\end{gathered}
$$

In view of (2.2) and (2.3), the operator equation

$$
L x=\lambda N x
$$

is equivalent to the following equations

$$
\begin{gathered}
x_{i}^{\prime}(t)=-\lambda a_{i}\left(t, x_{i}(t)\right)\left[b_{i}\left(t, x_{i}(t)\right)-\sum_{j=1}^{n} c_{i j}(t) f_{j}\left(x_{j}(t)\right)\right. \\
\left.-\sum_{j=1}^{n} d_{i j}(t) f_{j}\left(\int_{0}^{\infty} K_{i j}(u) x_{j}(t-u) d u\right)+I_{i}(t)\right], \\
i=1,2, \cdots, n, \lambda \in(0,1) . \quad(2.4)_{\lambda}
\end{gathered}
$$

Again from (2.2) and (2.3), it is not difficult to show that $\operatorname{Ker} L=R^{n}, \operatorname{Im} L=\{u(t)$ : $u(t)=\left(x_{1}(t), x_{2}(t), \cdots, x_{n}(t)\right)^{T} \in X, \int_{0}^{\omega} x_{1}(t) d t=$ $\left.\int_{0}^{\omega} x_{2}(t) d t=\cdots=\int_{0}^{\omega} x_{n}(t) d t=0\right\}$ is closed in $X$, $\operatorname{dimKer} L=n=\operatorname{codim} \operatorname{Im} L$, and $P, Q$ are continuous projectors such that

$$
\operatorname{ImP}=\operatorname{Ker} L \text { and } \operatorname{Ker} Q=\operatorname{Im} L .
$$

It follows that the operator $L$ is a Fredholm operator with index zero. Furthermore, the generalized inverse (of $L$ ) $K_{p}: \operatorname{Im} L \longrightarrow D o m L \cap \operatorname{Ker} P$ reads as

$$
\begin{gathered}
\left(K_{p} u\right)_{i}(t)=\int_{0}^{t} x_{i}(s) d s-\frac{1}{\omega} \int_{0}^{\omega} \int_{0}^{t} x_{i}(s) d s d t \\
u(t)=\left(x_{1}(t), x_{2}(t), \cdots, x_{n}(t)\right)^{T} \in \operatorname{ImL}
\end{gathered}
$$

for $i=1,2, \cdots, n$. Therefore, from (2.2) and (2.5), we have that $N$ is $L$-compact on $\bar{\Omega}$, where $\Omega$ is an open bounded set in $X$.

The following lemmas will be useful to prove our main results in Section 3-4.

Lemma 2.2.[24]-[25]. Let $H=\left(h_{i j}\right)_{n \times n}$ with $h_{i j} \leq 0, i, j=1,2, \cdots, n, i \neq j$. Then the following statements are equivalent.

(1) $H$ is an $M$-matrix.

(2) There exists a vector $\eta=\left(\eta_{1}, \eta_{2}, \cdots, \eta_{n}\right)$ $>(0,0, \cdots, 0)$ such that $\eta H>0$.

(3) There exists a vector $\xi=\left(\xi_{1}, \xi_{2}, \cdots, \xi_{n}\right)^{T}$ $>(0,0, \cdots, 0)^{T}$ such that $H \xi>0$.

Lemma 2.3 ([24]-[25]). Let $A \geq 0$ be an $n \times n$ matrix and $\rho(A)<1$, then $\left(E_{n}-A\right)^{-1} \geq 0$, where $E_{n}$ denotes the identity matrix of size $n$.

\section{Existence of a periodic solu- tion}

Theorem 3.1. Let $\left(H_{1}\right)-\left(H_{6}\right)$ hold. Assume that the following condition is satisfied .

$\left(H_{7}\right)$ there exists a vector $\eta=\left(\eta_{1}, \eta_{2}, \cdots, \eta_{n}\right)$ $>(0,0, \cdots, 0)$ such that

$$
\bar{\eta}=\left(\bar{\eta}_{1}, \bar{\eta}_{2}, \cdots, \bar{\eta}_{n}\right)=\eta\left(E_{n}-\Lambda\right)>(0,0, \cdots, 0),
$$

where $\Lambda=\left(q_{i j}\right)_{n \times n}, q_{i j}=\underline{b}_{i}^{-1}\left(\overline{c_{i j}} L_{j}+\overline{d_{i j}} L_{j} k_{i j}\right)(i, j$ $=1,2, \cdots, n)$. Then system (1.1) has at least one $\omega$-periodic solution.

Proof. We shall seek to apply Lemma 2.1. To do this, it suffices to prove that the set of all possible $\omega$-periodic solutions of Eq. $(2.4)_{\lambda}$ is bounded.

Let $u(t)=\left(x_{1}(t), x_{2}(t), \cdots, x_{n}(t)\right)^{T}$ be an arbitrary $\omega$ - periodic solution of Eq. (2.4) $\lambda$. Then, for any $i=1,2, \cdots, n, x_{i}(t)$, as the components of $u(t)$, are all continuously differentiable. Thus, there exist $t_{i} \in[0, \omega]$ such that $\left|x_{i}\left(t_{i}\right)\right|=\max _{t \in[0, \omega]}\left|x_{i}(t)\right|$. Hence, $x_{i}^{\prime}\left(t_{i}\right)=0, i=1,2, \cdots, n$. This implies that

$$
\begin{aligned}
& b_{i}\left(t_{i}, x_{i}\left(t_{i}\right)\right)=\sum_{j=1}^{n} c_{i j}\left(t_{i}\right) f_{j}\left(x_{j}\left(t_{i}\right)\right)+\sum_{j=1}^{n} d_{i j}\left(t_{i}\right) \\
& \times f_{j}\left(\int_{0}^{\infty} K_{i j}(u) x_{j}\left(t_{i}-u\right) d u\right)-I_{i}\left(t_{i}\right)
\end{aligned}
$$

In view of $\left(H_{1}\right)-\left(H_{6}\right)$, for $i=1,2, \cdots, n$, we have

$$
\begin{aligned}
& \underline{b}_{i}\left|x_{i}\left(t_{i}\right)\right| \leq\left|b_{i}\left(t_{i}, x_{i}\left(t_{i}\right)\right)\right| \\
\leq & \sum_{j=1}^{n}\left(\overline{c_{i j}} L_{j}+\overline{d_{i j}} L_{j} k_{i j}\right)\left|x_{j}\left(t_{j}\right)\right| \\
+ & \sum_{j=1}^{n}\left(\overline{c_{i j}}+\overline{d_{i j}}\right)\left|f_{j}(0)\right|+\left|I_{i}\left(t_{i}\right)\right|,
\end{aligned}
$$

Set $F_{i}=\sum_{j=1}^{n} \underline{b}_{i}^{-1}\left(\overline{c_{i j}}+\overline{d_{i j}}\right)\left|f_{j}(0)\right|+\underline{b}_{i}^{-1} \overline{I_{i}}, \quad i=$ $1,2, \cdots, n$. Clearly, (3.2) implies that

$$
\begin{aligned}
\left|x_{i}\left(t_{i}\right)\right| & \leq \sum_{j=1}^{n} \underline{b}_{i}^{-1}\left(\overline{c_{i j}} L_{j}+\overline{d_{i j}} L_{j} k_{i j}\right)\left|x_{j}\left(t_{j}\right)\right| \\
& +\sum_{j=1}^{n} \underline{b}_{i}^{-1}\left(\overline{c_{i j}}+\overline{d_{i j}}\right)\left|f_{j}(0)\right|+\underline{b}_{i}^{-1} \overline{I_{i}} \\
& =\sum_{j=1}^{n} q_{i j}\left|x_{j}\left(t_{j}\right)\right|+F_{i}, \quad i=1,2, \cdots, n .
\end{aligned}
$$

Thus,

$$
\left(E_{n}-\Lambda\right)\left(\left|x_{1}\left(t_{1}\right)\right|,\left|x_{2}\left(t_{2}\right)\right|, \cdots,\left|x_{n}\left(t_{n}\right)\right|\right)^{T} \leq
$$




$$
\left(F_{1}, F_{2}, \cdots, F_{n}\right)^{T}:=F,
$$

which, together with $\left(H_{7}\right)$, implies that

$$
\begin{aligned}
\min & \left\{\bar{\eta}_{1}, \bar{\eta}_{2}, \cdots, \bar{\eta}_{n}\right\}\left(\left|x_{1}\left(t_{1}\right)\right|+\left|x_{2}\left(t_{2}\right)\right|+\cdots+\left|x_{n}\left(t_{n}\right)\right|\right) \\
& \leq \bar{\eta}_{1}\left|x_{1}\left(t_{1}\right)\right|+\bar{\eta}_{2}\left|x_{2}\left(t_{2}\right)\right|+\cdots+\bar{\eta}_{n}\left|x_{n}\left(t_{n}\right)\right| \\
& =\eta\left(E_{n}-\Lambda\right)\left(\left|x_{1}\left(t_{1}\right)\right|,\left|x_{2}\left(t_{2}\right)\right|, \cdots,\left|x_{n}\left(t_{n}\right)\right|\right)^{T} \\
& \leq \eta\left(F_{1}, F_{2}, \cdots, F_{n}\right)^{T} \\
& =\eta_{1} F_{1}+\eta_{2} F_{2}+, \cdots,+\eta_{n} F_{n} .
\end{aligned}
$$

Therefore,

$$
\begin{gathered}
\left|x_{i}\right|_{\infty}=\max _{t \in[0, \omega]}\left|x_{i}(t)\right|=\left|x_{i}\left(t_{i}\right)\right| \leq \\
\frac{\eta_{1} F_{1}+\eta_{2} F_{2}+\cdots+\eta_{n} F_{n}}{\min \left\{\bar{\eta}_{1}, \bar{\eta}_{2}, \cdots, \bar{\eta}_{n}\right\}}:=\delta^{*}, i=1,2, \cdots, n .
\end{gathered}
$$

Again from $\left(H_{7}\right)$, it follows from Lemma 2.2 that $E_{n}-\Lambda$ is an $M$-matrix and there exist a vector $\zeta=\left(\zeta_{1}, \zeta_{2}, \cdots, \zeta_{2}\right)^{T}>(0,0, \cdots, 0)^{T}$ such that $\left(E_{n}-\Lambda\right) \zeta>(0,0, \cdots, 0)^{T}$, which implies that we can choose a constant $d>1$ such that $\bar{\xi}=$ $\left(\bar{\xi}_{1}, \bar{\xi}_{2}, \cdots, \bar{\xi}_{n}\right)^{T}=\left(d \zeta_{1}, d \zeta_{2}, \cdots, d \zeta_{n}\right)^{T}=d \zeta$ and $\xi_{i}=d \zeta_{i}>\delta^{*}, i=1,2, \cdots, n$, and

$$
\left(E_{n}-\Lambda\right) \bar{\xi}=d\left(E_{n}-\Lambda\right) \zeta>F .
$$

We take

$$
\Omega=\{u(t) \in X,-\bar{\xi}<u(t)<\bar{\xi}, \forall t \in R\},
$$

which satisfies Condition (1) of Lemma 2.1. If $u(t)=\left(x_{1}(t), x_{2}(t), \cdots, x_{n}(t)\right)^{T} \in \partial \Omega \cap K e r L$, then $u(t)$ is a constant vector in $R^{n}$, and there exists some $i \in\{1,2, \cdots, n\}$ such that $\left|x_{i}\right|=\bar{\xi}_{i}$. It follows that

$$
\begin{aligned}
& (Q N u)_{i}=-\frac{1}{\omega} \int_{0}^{\omega} a_{i}\left(t, x_{i}\right)\left[b_{i}\left(t, x_{i}\right)-\sum_{j=1}^{n} c_{i j}(t) f_{j}\left(x_{j}\right)\right. \\
& \left.-\sum_{j=1}^{n} d_{i j}(t) f_{j}\left(\int_{0}^{\infty} K_{i j}(u) x_{j}(t-u) d u\right)+I_{i}(t)\right] d t
\end{aligned}
$$

We claim that

$$
\left|(Q N u)_{i}\right|>0 \text {. }
$$

By way of contradiction, suppose that $\left|(Q N u)_{i}\right|=$ 0, i.e.,

$$
\begin{gathered}
-\frac{1}{\omega} \int_{0}^{\omega} a_{i}\left(t, x_{i}\right)\left[b_{i}\left(t, x_{i}\right)-\sum_{j=1}^{n} c_{i j}(t) f_{j}\left(x_{j}\right)\right. \\
\left.-\sum_{j=1}^{n} d_{i j}(t) f_{j}\left(\int_{0}^{\infty} K_{i j}(u) x_{j}(t-u) d u\right)+I_{i}(t)\right] d t=0 .
\end{gathered}
$$

Then, there exists some $t^{*} \in[0, \omega]$ such that

$$
\begin{gathered}
a_{i}\left(t^{*}, x_{i}\right)\left[b_{i}\left(t^{*}, x_{i}\right)-\sum_{j=1}^{n} c_{i j}\left(t^{*}\right) f_{j}\left(x_{j}\right)\right. \\
\left.-\sum_{j=1}^{n} d_{i j}\left(t^{*}\right) f_{j}\left(\int_{0}^{\infty} K_{i j}(u) x_{j}\left(t^{*}-u\right) d u\right)+I_{i}\left(t^{*}\right)\right]=0,
\end{gathered}
$$

which implies that

$$
\begin{gathered}
\underline{b}_{i}\left|x_{i}\right| \leq\left|b_{i}\left(t^{*}, x_{i}\right)\right|=\mid \sum_{j=1}^{n} c_{i j}\left(t^{*}\right) f_{j}\left(x_{j}\right) \\
+\sum_{j=1}^{n} d_{i j}\left(t^{*}\right) f_{j}\left(\int_{0}^{\infty} K_{i j}(u) x_{j}\left(t^{*}-u\right) d u\right)-I_{i}\left(t^{*}\right) \mid .
\end{gathered}
$$

Thus,

$$
\begin{aligned}
& \bar{\xi}_{i}=\left|x_{i}\right| \\
\leq & \sum_{j=1}^{n} \underline{b}_{i}^{-1}\left[\left|c_{i j}\left(t^{*}\right)\right|\left(\left|f_{j}\left(x_{j}\right)-f_{j}(0)\right|\right)\right. \\
+ & \left|d_{i j}\left(t^{*}\right)\right|\left(\left|f_{j}\left(\int_{0}^{\infty} K_{i j}(u) x_{j}\left(t^{*}-u\right) d u\right)-f_{j}(0)\right|\right) \\
+ & \left.\left(\left|c_{i j}\left(t^{*}\right)\right|+\left|d_{i j}\left(t^{*}\right)\right|\right)\left|f_{j}(0)\right|\right]+\underline{b}_{i}^{-1}\left|I_{i}\left(t^{*}\right)\right| \\
\leq & \sum_{j=1}^{n} q_{i j} \bar{\xi}_{j}+F_{i} .
\end{aligned}
$$

This implies that $\left(\left(E_{n}-\Lambda\right) \bar{\xi}\right)_{i} \leq F_{i}$, which contradicts $\left(E_{n}-\Lambda\right) \bar{\xi}>F$. Therefore, (3.10) holds, and hence, Condition (2) of Lemma 2.1 is satisfied.

Furthermore, we define a continuous function $\Psi: \Omega \bigcap \operatorname{KerL} \times[0,1] \longrightarrow X$ by $\Psi(u, \mu)=$ $\mu \operatorname{diag}\left(-\bar{a}_{1} \bar{b}_{1},-\bar{a}_{2} \bar{b}_{2}, \cdots,-\bar{a}_{n} \bar{b}_{n}\right) u+(1-\mu) Q N u$, for all $u=\left(x_{1}, x_{2}, \cdots, x_{n}\right)^{T} \in \Omega \bigcap \operatorname{KerL}=\Omega \bigcap R^{n}$ and $\mu \in[0,1]$.

If $u(t)=\left(x_{1}(t), x_{2}(t), \cdots, x_{n}(t)\right)^{T} \in \partial \Omega \cap$ $\operatorname{Ker} L$, then $u(t)$ is a constant vector in $R^{n}$, and there exists some $i \in\{1,2, \cdots, n\}$ such that $\left|x_{i}\right|=$ $\bar{\xi}_{i}$. It follows that

$$
\begin{gathered}
(\Psi(u, \mu))_{i}=-\mu \bar{a}_{i} \bar{b}_{i} x_{i}+(1-\mu)\left\{-\frac{1}{\omega} \int_{0}^{\omega} a_{i}\left(t, x_{i}\right)\right. \\
{\left[b_{i}\left(t, x_{i}\right)-\sum_{j=1}^{n} c_{i j}(t) f_{j}\left(x_{j}\right)-\sum_{j=1}^{n} d_{i j}(t) f_{j}\left(\int_{0}^{\infty} K_{i j}(u)\right.\right.} \\
\left.\left.\left.x_{j}(t-u) d u\right)+I_{i}(t)\right] d t\right\} .
\end{gathered}
$$

We claim that

$$
\left|(\Psi(u, \mu))_{i}\right|>0
$$


If this is not true, then $\left|(\Psi(u, \mu))_{i}\right|=0$, i.e.,

$$
\begin{gathered}
-\mu \bar{a}_{i} \bar{b}_{i} x_{i}+(1-\mu)\left\{-\frac{1}{\omega} \int_{0}^{\omega} a_{i}\left(t, x_{i}\right)\left[b_{i}\left(t, x_{i}\right)\right.\right. \\
-\sum_{j=1}^{n} c_{i j}(t) f_{j}\left(x_{j}\right)
\end{gathered}
$$

$\left.\left.-\sum_{j=1}^{n} d_{i j}(t) f_{j}\left(\int_{0}^{\infty} K_{i j}(u) x_{j}(t-u) d u\right)+I_{i}(t)\right] d t\right\}=0$.

Hence, there exists some $t^{* *} \in[0, \omega]$ such that

$$
\begin{gathered}
-\mu \bar{a}_{i} \bar{b}_{i} x_{i}+(1-\mu)\left\{-a_{i}\left(t^{* *}, x_{i}\right)\left[b_{i}\left(t^{* *}, x_{i}\right)\right.\right. \\
-\sum_{j=1}^{n} c_{i j}\left(t^{* *}\right) f_{j}\left(x_{j}\right)-\sum_{j=1}^{n} d_{i j}\left(t^{* *}\right) f_{j}\left(\int_{0}^{\infty} K_{i j}(u)\right. \\
\left.\left.\left.x_{j}\left(t^{* *}-u\right) d u\right)+I_{i}\left(t^{* *}\right)\right]\right\}=0 .
\end{gathered}
$$

Now, we shall consider the following two cases.

Case (i). If $x_{i}>0$, in view of $\left(H_{2}\right)$ and $\left(H_{3}\right)$,we get

$$
a_{i}\left(t^{* *}, x_{i}\right) b_{i}\left(t^{* *}, x_{i}\right)-\bar{a}_{i} \bar{b}_{i} x_{i} \leq 0 .
$$

Thus, from (3.13), we obtain

$$
\begin{gathered}
-a_{i}\left(t^{* *}, x_{i}\right) b_{i}\left(t^{* *}, x_{i}\right)+(1-\mu)\left\{-a_{i}\left(t^{* *}, x_{i}\right)\right. \\
{\left[-\sum_{j=1}^{n}\left(c_{i j}\left(t^{* *}\right) f_{j}\left(x_{j}\right)+d_{i j}\left(t^{* *}\right) f_{j}\left(\int_{0}^{\infty} K_{i j}(u)\right.\right.\right.} \\
\left.\left.\left.\left.\times x_{j}\left(t^{* *}-u\right) d u\right)\right)+I_{i}\left(t^{* *}\right)\right]\right\} \\
\geq \mu\left[a_{i}\left(t^{* *}, x_{i}\right) b_{i}\left(t^{* *}, x_{i}\right)-\bar{a}_{i} \bar{b}_{i} x_{i}\right]-a_{i}\left(t^{* *}, x_{i}\right) b_{i}\left(t^{* *}, x_{i}\right) \\
+(1-\mu)\left\{-a_{i}\left(t^{* *}, x_{i}\right)\left[-\sum_{j=1}^{n}\left(c_{i j}\left(t^{* *}\right) f_{j}\left(x_{j}\right)+d_{i j}\left(t^{* *}\right) \times\right.\right.\right. \\
\left.\left.\left.f_{j}\left(\int_{0}^{\infty} K_{i j}(u) x_{j}\left(t^{* *}-u\right) d u\right)\right)+I_{i}\left(t^{* *}\right)\right]\right\}=0,
\end{gathered}
$$

which implies that

$$
\begin{array}{ll} 
& a_{i}\left(t^{* *}, x_{i}\right) b_{i}\left(t^{* *}, x_{i}\right) \leq(1-\mu)\left\{-a_{i}\left(t^{* *}, x_{i}\right)\right. \\
\times \quad & {\left[-\sum_{j=1}^{n}\left(c_{i j}\left(t^{* *}\right) f_{j}\left(x_{j}\right)+I_{i}\left(t^{* *}\right)\right.\right.} \\
+ & \left.\left.\left.d_{i j}\left(t^{* *}\right) f_{j}\left(\int_{0}^{\infty} K_{i j}(u) x_{j}\left(t^{* *}-u\right) d u\right)\right)\right]\right\} \\
\leq & a_{i}\left(t^{* *}, x_{i}\right) \mid \sum_{j=1}^{n}\left(c_{i j}\left(t^{* *}\right) f_{j}\left(x_{j}\right)-I_{i}\left(t^{* *}\right)\right. \\
+ & \left.d_{i j}\left(t^{* *}\right) f_{j}\left(\int_{0}^{\infty} K_{i j}(u) x_{j}\left(t^{* *}-u\right) d u\right)\right) \mid .(3.1
\end{array}
$$

Noting that $x_{i}>0$ and $b_{i}\left(t^{* *}, x_{i}\right) \geq 0$, from (3.15), we have

$$
\begin{aligned}
& \underline{b}_{i} x_{i} \leq b_{i}\left(t^{* *}, x_{i}\right) \leq \mid \sum_{j=1}^{n}\left(c_{i j}\left(t^{* *}\right) f_{j}\left(x_{j}\right)+\right. \\
& \left.d_{i j}\left(t^{* *}\right) f_{j}\left(\int_{0}^{\infty} K_{i j}(u) x_{j}\left(t^{* *}-u\right) d u\right)\right)-I_{i}\left(t^{* *}\right) \mid .
\end{aligned}
$$

Therefore,

$$
\bar{\xi}_{i}=x_{i} \leq \sum_{j=1}^{n} q_{i j} \bar{\xi}_{j}+F_{i}
$$

This implies that $\left(\left(E_{n}-\Lambda\right) \bar{\xi}\right)_{i} \leq F_{i}$, which contradicts $\left(E_{n}-\Lambda\right) \bar{\xi}>F$. Therefore, (3.12) holds.

Case (ii). If $x_{i}<0$, using the methods similar to those used in Case (i), we can show that (3.12) holds. It follows that $\Psi\left(x_{1}, x_{2}, \cdots, x_{n}, \mu\right) \neq$ $(0, \quad 0, \cdots, \quad 0)^{T}, \quad \forall\left(x_{1}, \quad x_{2}, \cdots, x_{n}\right)^{T} \in$ $\partial \Omega \cap \operatorname{Ker} L, \mu \in[0,1]$.

Hence, using the homotopy invariance theorem, we obtain $\operatorname{deg}_{B}\left\{Q N, \Omega \cap \operatorname{KerL},(0,0, \cdots, 0)^{T}\right\}=$ $\operatorname{deg}_{B}\left\{\left(-\bar{a}_{1} \bar{b}_{1} x_{1},-\bar{a}_{2} \bar{b}_{2} x_{2}, \cdots,-\bar{a}_{n} \bar{b}_{n} x_{n}\right)^{T}, \Omega \cap \cap\right.$ $\left.\operatorname{Ker} L,(0,0, \cdots, 0)^{T}\right\} \neq 0$. To summarize, we have proved that $\Omega$ satisfies all the conditions of Lemma 2.1. This completes the proof.

\section{Uniqueness and exponential stability of the periodic solu- tion}

In this section, we establish some results for the uniqueness and exponential stability of the $\omega$-periodic solution of (1.1).

Theorem 4.1. Suppose that $\left(H_{1}\right)-\left(H_{7}\right)$ hold. Assume also that the following conditions are satisfied .

$\left(H_{8}\right)$ for $i=1,2, \ldots, n$, there exists a positive constant $L_{i}^{a}$ such that

$\left|a_{i}(t, u)-a_{i}(t, v)\right|<L_{i}^{a}|u-v|, \quad$ for all $t, u, v \in R$;

$\left(H_{9}\right)$ for $i=1,2, \ldots, n$, there exists a positive constant $L_{i}^{a b}$ such that

$$
\begin{gathered}
{\left[a_{i}(t, u) b_{i}(t, u)-a_{i}(t, v) b_{i}(t, v)\right](u-v) \geq 0,} \\
\left|a_{i}(t, u) b_{i}(t, u)-a_{i}(t, v) b_{i}(t, v)\right| \geq L_{i}^{a b}|u-v|,
\end{gathered}
$$

for all $t, u, v \in R$;

$\left(H_{10}\right)$ for $i=1,2, \ldots, n,\left|f_{j}(u)\right| \leq L_{j}|u|$ for all $u \in R$; 
$\left(H_{11}\right)$ for $i, j=1,2, \cdots, n$, set $\bar{F}_{i}=\underline{b}_{i}^{-1} \overline{I_{i}}, \delta=$ $\frac{\eta_{1} \bar{F}_{1}+\eta_{2} \bar{F}_{2}+\cdots+\eta_{n} \bar{F}_{n}}{\min \left\{\bar{\eta}_{1}, \bar{\eta}_{2}, \cdots, \bar{\eta}_{n}\right\}}$, and assume that $E_{n}-H$ is an $M$-matrix, where $H=\left(h_{i j}\right)_{n \times n}$ and

$$
\begin{gathered}
h_{i j}=\left[L_{i}^{a b}-L_{i}^{a} \sum_{j=1}^{n}\left(\overline{c_{i j}} L_{j} \delta+\overline{d_{i j}} L_{j} \delta k_{i j}\right)-L_{i}^{a} \overline{I_{i}}\right]^{-1} \\
\times \bar{a}_{i}\left(\overline{c_{i j}} L_{j}+\overline{d_{i j}} L_{j} k_{i j}\right), \\
L_{i}^{a b}-L_{i}^{a} \sum_{j=1}^{n}\left(\overline{c_{i j}} L_{j} \delta+\overline{d_{i j}} L_{j} \delta k_{i j}\right)-L_{i}^{a} \overline{I_{i}}>0 .
\end{gathered}
$$

Then system (1.1) has exactly one $\omega$-periodic solution, and the $\omega$-periodic solution of system (1.1) is globally exponentially stable.

Proof. Since $\left(H_{1}\right)-\left(H_{7}\right)$ hold, it follows from Theorem 3.1 that system (1.1) has at least one $\omega$ periodic solution $Z^{*}(t)=\left(x_{1}^{*}(t), x_{2}^{*}(t), \cdots, x_{n}^{*}(t)\right)^{T}$ with initial value $\varphi^{*}=\left(\varphi_{1}^{*}(t), \varphi_{2}^{*}(t), \cdots, \varphi_{n}^{*}(t)\right)^{T}$. from $\left(H_{10}\right)$, we have

$$
\left|f_{j}(0)\right|=0, \text { for } j=1,2, \ldots, n .
$$

Thus, by using a similar proof in (3.2) - (3.6), we obtain

$$
\begin{aligned}
\left|x_{i}^{*}\right|_{\infty} & =\max _{t \in[0, \omega]}\left|x_{i}^{*}(t)\right| \leq \frac{\eta_{1} F_{1}+\eta_{2} F_{2}+\cdots+\eta_{n} F_{n}}{\min \left\{\bar{\eta}_{1}, \bar{\eta}_{2}, \cdots, \bar{\eta}_{n}\right\}} \\
& =\frac{\eta_{1} \bar{F}_{1}+\eta_{2} \bar{F}_{2}+\cdots+\eta_{n} \bar{F}_{n}}{\min \left\{\bar{\eta}_{1}, \bar{\eta}_{2}, \cdots, \bar{\eta}_{n}\right\}}=\delta
\end{aligned}
$$

where $i=1,2, \cdots, n$.

Let $Z(t)=\left(x_{1}(t), x_{2}(t), \cdots, x_{n}(t)\right)^{T}$ be an arbitrary solution of system (1.1) with initial value $\varphi=\left(\varphi_{1}(t), \varphi_{2}(t), \cdots, \varphi_{n}(t)\right)^{T}$ and define $y(t)=$ $Z(t)-Z^{*}(t)$. Then, for $i=1,2, \cdots, n$, set

$$
\begin{gathered}
\alpha_{i}\left(t, y_{i}(t)\right)=a_{i}\left(t, y_{i}(t)+x_{i}^{*}(t)\right) b_{i}\left(t, y_{i}(t)+x_{i}^{*}(t)\right) \\
-a_{i}\left(t, x_{i}^{*}(t)\right) b_{i}\left(t, x_{i}^{*}(t)\right), \\
\beta_{i}\left(t, y_{i}(t)\right)=a_{i}\left(t, y_{i}(t)+x_{i}^{*}(t)\right) \sum_{j=1}^{n} c_{i j}(t)\left[f _ { j } \left(y_{j}(t)\right.\right. \\
\left.\left.+x_{j}^{*}(t)\right)-f_{j}\left(x_{j}^{*}(t)\right)\right], \\
\bar{\beta}_{i}\left(t, y_{i}(t)\right)=a_{i}\left(t, y_{i}(t)+x_{i}^{*}(t)\right) \sum_{j=1}^{n} d_{i j}(t) \\
\times\left[f _ { j } \left(\int_{0}^{\infty} K_{i j}(u)\left(y_{j}(t-u)+x_{j}^{*}(t-u) d u\right)\right.\right. \\
-f_{j}\left(\int_{0}^{\infty} K_{i j}(u)\left(x_{j}^{*}(t-u) d u\right)\right] \\
\gamma_{i}\left(t, y_{i}(t)\right)=\left[a_{i}\left(t, y_{i}(t)+x_{i}^{*}(t)\right)\right.
\end{gathered}
$$

$$
\begin{gathered}
\left.-a_{i}\left(t, x_{i}^{*}(t)\right)\right] \sum_{j=1}^{n} c_{i j}(t) f_{j}\left(x_{j}^{*}(t)\right), \\
\bar{\gamma}_{i}\left(t, y_{i}(t)\right)=\left[a_{i}\left(t, y_{i}(t)+x_{i}^{*}(t)\right)-a_{i}\left(t, x_{i}^{*}(t)\right)\right] \\
\times \sum_{j=1}^{n} d_{i j}(t) f_{j}\left(\int_{0}^{\infty} K_{i j}(u) x_{j}^{*}(t-u) d u\right), \\
\theta_{i}\left(t, y_{i}(t)\right)=\left[a_{i}\left(t, y_{i}(t)+x_{i}^{*}(t)\right)-a_{i}\left(t, x_{i}^{*}(t)\right)\right] I_{i}(t) .
\end{gathered}
$$

Then, we get

$$
\begin{aligned}
& y_{i}^{\prime}(t)=-\alpha_{i}\left(t, y_{i}(t)\right)+\beta_{i}\left(t, y_{i}(t)\right)+\bar{\beta}_{i}\left(t, y_{i}(t)\right) \\
& +\gamma_{i}\left(t, y_{i}(t)\right)+\bar{\gamma}_{i}\left(t, y_{i}(t)\right)-\theta_{i}\left(t, y_{i}(t)\right),
\end{aligned}
$$

Thus, for $i=1,2, \cdots, n$, from $\left(H_{2}\right),\left(H_{4}\right),\left(H_{5}\right)$, $\left(H_{8}\right),\left(H_{9}\right)$ and $\left(H_{10}\right)$, we have

$$
\begin{array}{ll}
D^{-} & \left|y_{i}(t)\right|=D^{-}\left\{\operatorname{sgn}\left(x_{i}(t)-x_{i}^{*}\right)\left(x_{i}(t)-x_{i}^{*}\right)\right\} \\
\leq \quad & -L_{i}^{a b}\left|y_{i}(t)\right|+\left|\beta_{i}\left(t, y_{i}(t)\right)\right|+\left|\bar{\beta}_{i}\left(t, y_{i}(t)\right)\right| \\
& +\left|\gamma_{i}\left(t, y_{i}(t)\right)\right|+\left|\bar{\gamma}_{i}\left(t, y_{i}(t)\right)\right|+\left|\theta_{i}\left(t, y_{i}(t)\right)\right| \\
\leq \quad & -\left[L_{i}^{a b}-L_{i}^{a} \sum_{j=1}^{n}\left(\overline{c_{i j}} L_{j} \delta+\overline{d_{i j}} L_{j} k_{i j} \delta\right)-\right. \\
& \left.L_{i}^{a} \overline{I_{i}}\right]\left|y_{i}(t)\right|+\bar{a}_{i} \sum_{j=1}^{n}\left(\overline{c_{i j}} L_{j}+\overline{d_{i j}} L_{j} k_{i j}\right) \overline{y_{j}}(t),
\end{array}
$$

where $\bar{y}_{j}(t)=\sup _{-\infty<s \leq t}\left|y_{j}(s)\right|$. From $\left(H_{9}\right)$ and Lemma 2.2, we obtain that there exist a constant $\sigma>0$ and a vector $\xi=\left(\xi_{1}, \xi_{2}, \cdots, \xi_{n}\right)^{T}>$ $(0,0, \cdots, 0)^{T}$ such that

$$
\left(E_{n}-H\right) \xi>(\sigma, \sigma, \cdots, \sigma)^{T} .
$$

Therefore, for $i=1,2, \cdots, n$,

$$
\begin{gathered}
\xi_{i}-\sum_{j=1}^{n} k_{i j} \xi_{j}=\xi_{i}-\sum_{j=1}^{n}\left[L_{i}^{a b}-L_{i}^{a} \sum_{j=1}^{n}\left(\overline{c_{i j}} L_{j} \delta\right.\right. \\
\left.\left.+\overline{d_{i j}} L_{j} \delta k_{i j}\right)-L_{i}^{a} \overline{I_{i}}\right]^{-1} \bar{a}_{i}\left(\overline{c_{i j}} L_{j}+\overline{d_{i j}} L_{j} k_{i j}\right) \xi_{j}>\sigma,
\end{gathered}
$$

which implies that

$$
\begin{gathered}
-\left[L_{i}^{a b}-L_{i}^{a} \sum_{j=1}^{n}\left(\overline{c_{i j}} L_{j} \delta+\overline{d_{i j}} L_{j} \delta k_{i j}\right)-L_{i}^{a} \overline{I_{i}}\right] \xi_{i} \\
+\bar{a}_{i} \sum_{j=1}^{n}\left(\overline{c_{i j}} L_{j}+\overline{d_{i j}} L_{j} k_{i j}\right) \xi_{j}<0,
\end{gathered}
$$

where $i=1,2, \cdots, n$. We can choose a constant $\alpha<1$ such that

$\alpha \xi_{i}+\left\{-\left[L_{i}^{a b}-L_{i}^{a} \sum_{j=1}^{n}\left(\overline{c_{i j}} L_{j} \delta+\overline{d_{i j}} L_{j} \delta k_{i j}\right)-L_{i}^{a} \overline{I_{i}}\right] \xi_{i}\right.$ 


$$
\left.+\bar{a}_{i} \sum_{j=1}^{n}\left(\overline{c_{i j}} L_{j}+\overline{d_{i j}} L_{j} k_{i j}\right) \xi_{j} e^{\alpha \tau}\right\}<0,
$$

We can also choose a constant $\beta>1$ such that $\beta \xi_{i} e^{-\alpha t}>1$, for all $t \in(-\infty, 0], i=1,2, \cdots, n$.

For $\forall \varepsilon>0$, let

$$
\Upsilon_{i}(t)=\beta \xi_{i}\left[\sum_{j=1}^{n} \overline{y_{j}}(0)+\varepsilon\right] e^{-\alpha t}, i=1,2, \cdots, n .
$$

From (4.6) and (4.8), we obtain

$$
\begin{gathered}
D_{-} \Upsilon_{i}(t)=-\alpha \beta \xi_{i}\left[\sum_{j=1}^{n} \overline{y_{j}}(0)+\varepsilon\right] e^{-\alpha t} \\
>\quad-\left[L_{i}^{a b}-2 L_{i}^{a} \sum_{j=1}^{n}\left(\overline{c_{i j}} L_{j} \delta+\overline{d_{i j}} L_{j} \delta k_{i j}\right)-L_{i}^{a} \overline{I_{i}}\right] \\
\Upsilon_{i}(t)+\bar{a}_{i} \sum_{j=1}^{n}\left(\overline{c_{i j}} L_{j}+\overline{d_{i j}} L_{j} k_{i j}\right) \bar{\Upsilon}_{j}(t)
\end{gathered}
$$

where $\bar{\Upsilon}_{j}(t)=\sup _{-\infty<s \leq 0} \Upsilon_{j}(s)$. In view of (4.7) and (4.8), for $i=1,2, \cdots, n$, we have

$$
\begin{aligned}
& \Upsilon_{i}(t)=\beta \xi_{i}\left[\sum_{j=1}^{n} \overline{y_{j}}(0)+\varepsilon\right] e^{-\alpha t}>\sum_{j=1}^{n} \overline{y_{j}}(0) \\
& +\varepsilon>\left|y_{i}(t)\right|, \text { for all } t \in(-\infty, 0] .
\end{aligned}
$$

We claim that

$$
\left|y_{i}(t)\right|<\Upsilon_{i}(t) \text {, for all } t>0,
$$

Contrarily, there must exist $i \in\{1,2, \cdots, n\}$ and $t_{i}>0$ such that

$$
\left|y_{i}\left(t_{i}\right)\right|=\Upsilon_{i}\left(t_{i}\right) \text { and }\left|y_{j}(t)\right|<\Upsilon_{j}(t),
$$

for all $t \in\left[-\tau, t_{i}\right), j=1,2, \cdots, n$,

which implies that

$$
\begin{aligned}
& \left|y_{i}\left(t_{i}\right)\right|-\Upsilon_{i}\left(t_{i}\right)=0 \text { and }\left|y_{j}(t)\right|-\Upsilon_{j}(t)<0, \\
& \text { for all } t \in\left[-\tau, t_{i}\right), j=1,2, \cdots, n .
\end{aligned}
$$

It follows that

$$
\begin{aligned}
0 \leq & D^{-}\left(\left|y_{i}\left(t_{i}\right)\right|-\Upsilon_{i}\left(t_{i}\right)\right) \\
\leq & \limsup _{h \longrightarrow 0^{-}} \frac{\left|y_{i}\left(t_{i}+h\right)\right|-\left|y_{i}\left(t_{i}\right)\right|}{h} \\
& -\liminf _{h \longrightarrow 0^{-}} \frac{\Upsilon_{i}\left(t_{i}+h\right)-\Upsilon_{i}\left(t_{i}\right)}{h} \\
= & D^{-}\left|y_{i}\left(t_{i}\right)\right|-D_{-} \Upsilon_{i}\left(t_{i}\right) .
\end{aligned}
$$

From (4.4), (4.9) and (4.12), we obtain

$$
\begin{gathered}
D^{-}\left|y_{i}\left(t_{i}\right)\right| \\
\leq \quad-\left[L_{i}^{a b}-L_{i}^{a} \sum_{j=1}^{n}\left(\overline{c_{i j}} L_{j} \delta+\overline{d_{i j}} L_{j} \delta k_{i j}\right)-L_{i}^{a} \overline{I_{i}}\right] \\
\left|y_{i}\left(t_{i}\right)\right|+\bar{a}_{i} \sum_{j=1}^{n}\left(\overline{c_{i j}} L_{j}+\overline{d_{i j}} L_{j} k_{i j}\right) \overline{y_{j}}\left(t_{i}\right) \\
\leq \quad-\left[L_{i}^{a b}-L_{i}^{a} \sum_{j=1}^{n}\left(\overline{c_{i j}} L_{j} \delta+\overline{d_{i j}} L_{j} \delta k_{i j}\right)-L_{i}^{a} \overline{I_{i}}\right] \\
\quad \Upsilon_{i}\left(t_{i}\right)+\bar{a}_{i} \sum_{j=1}^{n}\left(\overline{c_{i j}} L_{j}+\overline{d_{i j}} L_{j} k_{i j}\right) \bar{\Upsilon}_{j}\left(t_{i}\right) \\
<\quad D_{-} \Upsilon_{i}\left(t_{i}\right),
\end{gathered}
$$

which contradicts (4.14). Hence, (4.11) holds. Letting $\varepsilon \longrightarrow 0^{+}$and $M=n \max _{1 \leq i \leq n}\left\{\beta \xi_{i}+1\right\}$, we have from (4.8) and (4.11) that

$$
\begin{gathered}
\left|x_{i}(t)-x_{i}^{*}(t)\right|=\left|y_{i}(t)\right| \leq \beta \xi_{i} \sum_{j=1}^{n} \overline{y_{j}}(0) e^{-\alpha t} \\
\leq \beta \xi_{i} n\left\|\varphi-\varphi^{*}\right\| e^{-\alpha t} \leq M\left\|\varphi-\varphi^{*}\right\| e^{-\alpha t},
\end{gathered}
$$

for all $t>0$. This completes the proof.

\section{An illustrative example}

Example 5.1. Consider the following GCNNs

$$
\left\{\begin{array}{c}
x_{1}^{\prime}(t)=-\left(2+\frac{1}{10 \pi} \arctan x_{1}(t)\right)\left[x_{1}(t)\right. \\
\quad-\frac{1}{24}(\sin t)\left|x_{1}(t)\right|-\frac{1}{24}(\cos t)\left|x_{2}(t)\right| \\
\quad-\frac{1}{24}(\sin t)\left|\int_{0}^{\infty}(\sin u) e^{-u} x_{1}(t-u) d u\right| \\
\quad-\frac{1}{24}(\cos t)\left|\int_{0}^{\infty}(\sin u) e^{-u} x_{2}(t-u) d u\right| \\
\left.\quad+\frac{5}{12} \sin ^{2} t\right] \\
x_{2}^{\prime}(t)=-\left(2+\frac{1}{10 \pi} \arctan x_{2}(t)\right)\left[x_{2}(t)-\right. \\
\quad \frac{1}{24}(\sin 2 t)\left|x_{1}(t)\right|-\frac{1}{24}(\cos 4 t)\left|x_{2}(t)\right|- \\
\quad \frac{1}{24}(\sin 2 t)\left|\int_{0}^{\infty}(\sin u) e^{-u} x_{1}(t-u) d u\right|- \\
\quad \frac{1}{24}(\cos 4 t)\left|\int_{0}^{\infty}(\sin u) e^{-u} x_{2}(t-u) d u\right| \\
\quad+\frac{5}{12} \cos t .
\end{array}\right.
$$

Notice that $f_{1}(x)=f_{2}(x)=|x|, K_{i j}(u)=$ $(\sin u) e^{-u}, a_{1}(t, x)=a_{2}(t, x)=2+\frac{1}{10 \pi} \arctan x$, $b_{1}(t, x)=b_{2}(t, x)=x$, it is easy to show that we can choose the constants in the conditions $\left(H_{1}\right)-\left(H_{10}\right)$ and $\left(H_{11}^{*}\right)$ as follows.

$\underline{a}_{1}=\underline{a}_{2}=1, \bar{a}_{1}=\bar{a}_{2}=3, \bar{b}_{1}=\bar{b}_{2}=\bar{b}_{1}=\bar{b}_{2}=$ $1, L_{1}=L_{2}=1, L_{1}^{a}=L_{2}^{a}=\frac{1}{30}, L_{1}^{a b}=L_{2}^{a b}=1, \bar{I}_{1}=$ $\bar{I}_{2}=\frac{5}{12}, \overline{c_{11}}=\overline{d_{11}}=\overline{c_{12}}=\overline{c_{12}}=\overline{c_{21}}=\overline{d_{21}}=\overline{c_{22}}=$ $\overline{d_{22}}=\frac{1}{24}, \eta=\left(\eta_{1}, \eta_{2}\right)=(1,1), k_{i j}=1, i, j=1,2$. Then, we obtain

$$
\bar{\eta}=\left(\bar{\eta}_{1}, \bar{\eta}_{2}\right)=\eta\left(E_{n}-\Lambda\right)=\left(\frac{5}{6}, \frac{5}{6}\right), \quad \delta=1
$$




$$
L_{i}^{a b}-L_{i}^{a} \sum_{j=1}^{2}\left(\overline{c_{i j}} L_{j} \delta+\overline{d_{i j}} L_{j} \delta k_{i j}\right)-L_{i}^{a} \overline{I_{i}}=\frac{353}{360},
$$

$\mathrm{i}=1,2$, and

$$
H=\left(h_{i j}\right)_{2 \times 2}=\left(\begin{array}{cc}
\frac{90}{353} & \frac{90}{353} \\
\frac{90}{353} & \frac{90}{353}
\end{array}\right),
$$

which implies that

$$
\rho(H) \leq\|H\|_{\infty}=\frac{90}{353}+\frac{90}{353}=\frac{180}{353}<1,
$$

where $\|H\|_{\infty}$ the row norm of matrix $H$. It is straight forward to check that all the conditions needed in Corollary 4.1 are satisfied. Therefore, by Corollary 4.1, system (5.1) has exactly one $2 \pi$ periodic solution, and the $2 \pi$-periodic solution of system (5.1) is globally exponentially stable.

\section{Conclusion}

In this paper, Cohen-Grossberg neural networks with continuously distributed delays have been studied. Some sufficient conditions for the existence and exponential stability of the periodic solutions have been established. These obtained results are new. Moreover, an example is given to illustrate the effectiveness of the new results.

\section{Acknowledgements}

This work is partially supported by National Nature Science Foundation of China (Grant No. 60674020).

\section{References}

[1] M. Cohen, S. Grossberg, Absolute stability and global pattern formation and parallel memory storage by competiticve neural networks. IEEE Trans. Man Cybernet., 13: 815-826, 1983.

[2] K. Gopalsamy, X. Z. He, Delay-independent stability in bidirection associative memory networks. IEEE Trans. Neural Networks, 5: 998-1002, 1994.

[3] X. Li, L. Huang, H. Zhu, Global stability of cellular neural networks with constant and variable delays. Nonlinear Anal., 53: 319-333, 2003.

[4] S. Guo, L. Huang, Stability analysis of a delayed Hopfield neural network. Physical Review E, 67 : 061902, 2003.

[5] J. Cao, Global stability analysis in delayed cellular neural networks. Phys. Rev. E, 59: 5940-5944, 1999.

[6]L. Wang, X. Zou, Harmless delays in Cohen-Grossberg neural networks. Physics D, 170: 162-173, 2002.
[7] J. Cao, J. Liang, Boundedness and stability for CohenGrossberg neural networks with time-varing delays. $J$. Math. Anal. Appl., 296: 665-685, 2004.

[8] T. Chen, L. Rong, Delay-independent stability analysis of Cohen-Grossberg neural networks. Physics Letters A, 317: 436-449, 2003.

[9] Y. Li, Existence and stability of periodic solutions for Cohen-Grossberg neural networks with multiple delays. Chaos, Solutions and Fractals, 20: 459-466, 2004.

[10] X. Liao, C. Li and K. Wong, Criteria for exponential stability of Cohen-Grossberg neural networks. Neural Networks, 17: 1401-1414, 2004.

[11] L. Wang, Stability of Cohen-Grossberg neural networks with distributed delays. Appl. Math. Comput., 160: 93-110, 2005.

[12] L. Wang, X. Zou, Exponential stability of CohenGrossberg neural networks. Neural Networks, 15: 415422, 2002

[13] L. F. Shampine, S. Thompson, Solving DDEs in Matlab. Applied Numerical Mathematics. 37: 441-458, 2001.

[14] Tiejun Zhou, Anping Chen and Yuyuan Zhou, Existence and global exponential stability of periodic solution to BAM neural networks with periodic coefficients and continuously distributed delays. Phys.Lett. A, 343(5): 336-350, 2005.

[15] Qiang Zhang, Xiaopeng Wei and Jin Xu , Global exponential stability of Hopfield neural networks with continuously distributed delays. Phys.Lett. A, 315(6): 431-436, 2003.

[16] Zhang Qiang, MA Run-Nian, and XU Jin, Global Exponential Convergence Analysis of Hopfield Neural Networks with Continuously Distributed Delays. Communications in Theoretical Physics, 39(3):381-384, 2003.

[17] Zhang Qiang, MA Run-Nian, and XU Jin, Global stability of bidirectional associative memory neural networks with continuously distributed delays. Science in China, Ser.F, 46(5): 327-334, 2003.

[18]Yixuan Wang, Wanmin Xiong, Qiyuan Zhou, Bing Xiao and Yuehua Yu, Global exponential stability of cellular neural networks with continuously distributed delays and impulses. Phys.Lett. A, 350(1-2): 89-95, 2006.

[19] Z. Liu, L. Liao, Existence and global exponential stability of periodic solution of cellular neural networks with time-varying delays. J. Math. Anal. Appl., 290: 247-262, 2004.

[20] M. Morita, Associative memory with nonmonotone dynamics. Neural Networks, 6: 115-126, 1993.

[21]K.Gopalsamy, X.Z. He, Stability in asymmetric Hopfield nets with transmission delays. Phys. D, 76:344358, 1994.

[22]M. P. Kennedy and L. O. Chua, Neural networks for nonlinear programming. IEEE Trans. Syst., 35: 554$562,1988$.

[23] R. E. Gaines and J. L. Mawhin, Coincidence Degree, and Nonlinear Differential Equations, Springer-Verlag, Berlin, 1977.

[24] J. P. LaSalle, The Stability of Dynamical System, SIAM, Philadelphia, 1976.

[25] A. Berman, R. J. Plemmons, Nonnegative Matrices in the Mathematical Science, Academic Press, New York, 1979. 